PROCEEDINGS OF THE

AMERICAN MATHEMATICAL SOCIETY

Volume 132, Number 4, Pages 1059-1066

S 0002-9939(03)07139-9

Article electronically published on July 14, 2003

\title{
ON INJECTIVE OR DENSE-RANGE OPERATORS LEAVING A GIVEN CHAIN OF SUBSPACES INVARIANT
}

\author{
BAMDAD R. YAHAGHI
}

(Communicated by Joseph A. Ball)

With gratitude, dedicated to H. Hajiabolhassan, I. Mirfazeli, and F. Nouri

\begin{abstract}
In this paper we prove the existence of dense-range or one-to-one compact operators on a separable Banach space leaving a given finite chain of subspaces invariant. We use this result to prove that a semigroup $\mathcal{S}$ of bounded operators is reducible if and only if there exists an appropriate oneto-one compact operator $K$ such that the collection $\mathcal{S} K$ of compact operators is reducible.
\end{abstract}

\section{Introduction and Preliminary Results}

We start by recalling some definitions and standard notation. Throughout this paper, unless otherwise stated, $\mathcal{X}$ stands for a separable real or complex Banach space. As is usual, by $\mathbb{F}$ we mean $\mathbb{R}$ or $\mathbb{C}$. The term subspace will be used to describe a closed subspace of a Banach space $X$. The subspaces $\{0\}$ and $X$ are called the trivial subspaces of $\mathcal{X} ; \mathcal{B}(X)$ denotes the set (in fact the algebra) of bounded operators on $X ; \mathcal{B}_{0}(X)$ is used to denote the set (in fact the ideal) of compact operators on $\mathcal{X}$. A subspace $\mathcal{M}$ is invariant for a collection $\mathcal{F}$ of bounded operators if $T \mathcal{M} \subseteq \mathcal{M}(T \in \mathcal{F})$. A collection $\mathcal{F}$ of bounded operators on a space of dimension greater than one is called reducible if it has a nontrivial invariant subspace. In case the dimension of the underlying space is one or zero, then the collection $\mathcal{F}$ is called reducible if $\mathcal{F}=\{0\}$ or $\mathcal{F}=\emptyset$, respectively, where $\emptyset$ denotes the empty set. For a collection $\mathcal{C}$ of vectors, $\langle\mathcal{C}\rangle$ is used to denote the (not necessarily closed) linear manifold spanned by $\mathrm{C}$.

We start off with a well-known lemma.

Lemma 1.1. Let $f, f_{j}(1 \leq j \leq n)$ be linear functionals on a vector space $\mathcal{V}$. Then $f$ is a linear combination of $f_{1}, \ldots, f_{n}$, i.e., $f \in\left\langle f_{j}\right\rangle_{j=1}^{n}$, iff $\bigcap_{j=1}^{n} \operatorname{Ker} f_{j} \subseteq \operatorname{Ker} f$.

Proof. See [M], page 78 .

Corollary 1.2. Let $f, f_{j}(1 \leq j \leq n)$ be linear functionals on a vector space $\mathcal{V}$, and let $\mathcal{W}$ be a subspace of $\mathcal{V}$. Then $f \in\left\langle f_{1}, \ldots, f_{n}, \mathcal{W}^{\perp}\right\rangle$ iff $\mathcal{W} \cap\left(\bigcap_{j=1}^{n} \operatorname{Ker} f_{j}\right) \subseteq \operatorname{Ker} f$. $\left(\right.$ Here $\left.\mathcal{W}^{\perp}:=\left\{f \in \mathcal{V}^{\prime}: f(\mathcal{W})=0\right\}.\right)$

Received by the editors October 15, 2002 and, in revised form, November 16, 2002.

2000 Mathematics Subject Classification. Primary 47A15, 47A46, 47D03.

Key words and phrases. Linear functional, invariant subspace, reducible, weak* topology.

The author gratefully acknowledges the support of an Izaak Walton Killam Memorial Scholarship at Dalhousie University as well as an NSERC PDF at the University of Toronto. 


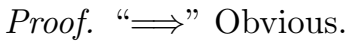

" $\Longleftarrow "$ Let $g, g_{j}(1 \leq j \leq n)$ be, respectively, the restrictions of $f, f_{j}(1 \leq j \leq n)$ to the subspace $\mathcal{W}$. It easily follows from the hypothesis that

$$
\bigcap_{j=1}^{n} \operatorname{Ker} g_{j} \subseteq \operatorname{Ker} g .
$$

So by Lemma $1.1 g \in\left\langle g_{j}\right\rangle_{j=1}^{n}$. Thus there are scalars $c_{j}(1 \leq j \leq n)$, such that $g=\sum_{j=1}^{n} c_{j} g_{j}$. Write

$$
f=\left(f-\sum_{j=1}^{n} c_{j} f_{j}\right)+\sum_{j=1}^{n} c_{j} f_{j}=f^{\prime}+\sum_{j=1}^{n} c_{j} f_{j},
$$

where $f^{\prime}=f-\sum_{j=1}^{n} c_{j} f_{j}$. It is plain that

$$
f^{\prime}(\mathcal{W})=\left(f-\sum_{j=1}^{n} c_{j} f_{j}\right)(\mathcal{W})=\left(g-\sum_{j=1}^{n} c_{j} g_{j}\right)(\mathcal{W})=0 .
$$

Hence $f^{\prime} \in \mathcal{W}^{\perp}$. It follows that $f \in\left\langle f_{1}, \ldots, f_{n}, \mathcal{W}^{\perp}\right\rangle$.

Another preliminary result that we need is the following.

Proposition 1.3. Let $\mathcal{X}$ be a separable Banach space. Then $X^{*}$ is $w^{*}$-separable.

Proof. Clearly $X^{*}=\bigcup_{n=1}^{\infty} B_{n}$ where $B_{n}$ is the closed ball of radius $n(n \in \mathbb{N})$ in $X^{*}$. By the Banach-Alaoglu Theorem, every $B_{n}$ is $\mathrm{w}^{*}$-compact and by Theorem 5.5.1 of C] every $B_{n}$ is w*-metrizable, implying that every $B_{n}$ is w*-separable. Now since every $B_{n}$ is w*-separable, so is $X^{*}=\bigcup_{n=1}^{\infty} B_{n}$.

\section{An Existence Result}

In this section, for a given subspace of a Banach space we prove the existence of certain types of compact operators leaving the given subspace invariant. Then we use this fact to obtain a necessary and sufficient condition for reducibility of a semigroup of bounded operators.

Theorem 2.1. Let $\mathcal{V}$ be a subspace of an infinite-dimensional separable Banach space $X$.

(a) There exists a compact operator $K$ in the norm closure of finite-rank operators on $X$ whose range is dense and such that $\overline{K \mathcal{V}}=\mathcal{V}$.

(b) There exists a one-to-one compact operator $K$ in the norm closure of finiterank operators on $\mathcal{X}$ that leaves $\mathcal{V}$ invariant.

Remark. It is worth mentioning that for (real or complex) Hilbert spaces we can obtain stronger results: there exists a one-to-one compact normal operator $K$ whose range is dense and such that $\overline{K \mathcal{V}}=\mathcal{V}$. To see this, find an orthonormal basis for the given subspace $\mathcal{V}$ and extend the orthonormal basis to an orthonormal basis $\mathcal{B}$ for $\mathcal{X}$, and let $K$ be a diagonal compact normal operator relative to $\mathcal{B}$. It is plain that $K$ has dense range and that $\overline{K V}=\mathcal{V}$. Now since $K$ is normal and has dense range, it follows that $K$ is one-to-one, which is what we wanted.

Proof. (a) Let $\left\{x_{i}\right\}_{i=1}^{\infty}$ be a dense subset of $\mathcal{V}$. Enlarge $\left\{x_{i}\right\}_{i=1}^{\infty}$ to a dense subset $\left\{x_{i}\right\}_{i=1}^{\infty} \cup\left\{y_{i}\right\}_{i=1}^{\infty}$ of $\mathcal{X}$. Without loss of generality, we may assume that $x_{i} \neq 0, y_{i} \notin$ $\mathcal{V}$ for all $i \in \mathbb{N}$. If necessary, by choosing subsequences of $\left\{x_{i}\right\}_{i=1}^{\infty}$ and $\left\{y_{i}\right\}_{i=1}^{\infty}$, 
we may suppose that $\left\{x_{i}\right\}_{i=1}^{\infty} \cup\left\{y_{i}\right\}_{i=1}^{\infty}$ is independent, $\overline{\left\langle\left\{x_{i}\right\}_{i=1}^{\infty}\right\rangle}=\mathcal{V}$, and that $\overline{\left\langle\left\{x_{i}\right\}_{i=1}^{\infty} \cup\left\{y_{i}\right\}_{i=1}^{\infty}\right\rangle}=X$.

Let $f_{1}$ be any functional such that $f_{1}\left(x_{1}\right) \neq 0$. Set $T_{1}:=x_{1} \otimes f_{1}$ defined by $T_{1}(x)=f_{1}(x) x_{1}$. By the Hahn-Banach Theorem there is a linear functional $f_{2}$ such that $f_{2}\left(x_{1}\right)=0$ but $f_{2}\left(x_{2}\right) \neq 0$. Define $T_{2}:=x_{2} \otimes f_{2}$. Suppose that $f_{j}$ 's $(1 \leq j \leq k-1)$ are chosen. Since $\left\{x_{i}\right\}_{i=1}^{k}$ is independent, by the Hahn-Banach Theorem there is a functional $f_{k}$ such that $f_{k}(x)=0$ for all $x \in\left\langle x_{1}, \ldots, x_{k-1}\right\rangle$ but $f_{k}\left(x_{k}\right) \neq 0$. Define $T_{k}:=x_{k} \otimes f_{k}$. Note that $T_{k}\left(x_{j}\right)=f_{k}\left(x_{j}\right) x_{k}$ and hence $T_{k}\left(x_{j}\right)=0$ for $k>j$ and $T_{k}\left(x_{k}\right)=f_{k}\left(x_{k}\right) x_{k} \neq 0$.

Now, again by the Hahn-Banach Theorem, there is a linear functional $f_{1}^{\prime}$ such that $f_{1}^{\prime}(x)=0$ for all $x \in \mathcal{V}$ but $f_{1}^{\prime}\left(y_{1}\right) \neq 0$. Define $T_{1}^{\prime}:=y_{1} \otimes f_{1}^{\prime}$. Suppose that $f_{j}^{\prime}$ 's $(1 \leq j \leq k-1)$ are chosen. So by the Hahn-Banach Theorem there is a functional $f_{k}^{\prime}$ such that $f_{k}^{\prime}(x)=0$ for all $x \in\left\langle y_{1}, \ldots, y_{k-1}, \mathcal{V}\right\rangle$ but $f_{k}^{\prime}\left(y_{k}\right) \neq 0$. Set $T_{k}^{\prime}:=y_{k} \otimes f_{k}^{\prime}$. Note that $T_{k}^{\prime}(x)=0$ for all $x \in \mathcal{V}$. In particular, $T_{k}^{\prime}\left(x_{j}\right)=0$ for all positive integers $j$ and $k$. Also note that $T_{k}^{\prime} y_{j}=f_{k}^{\prime}\left(y_{j}\right) y_{k}$, and hence $T_{k}^{\prime} y_{j}=0$ for $k>j$ and $T_{k}^{\prime} y_{k}=f_{k}^{\prime}\left(y_{k}\right) y_{k} \neq 0$.

Now define

$$
K:=\sum_{j=1}^{\infty}\left(\frac{T_{j}}{2^{j}\left\|T_{j}\right\|}+\frac{T_{j}^{\prime}}{2^{j}\left\|T_{j}^{\prime}\right\|}\right) .
$$

That $K$ is well-defined is obvious since the series defining $K$ is absolutely convergent, hence convergent. We need to show that $K$ is compact, has dense range, and that $\overline{K V}=\mathcal{V}$. That $K$ is compact is obvious because $K$ is the norm limit of finite rank, hence compact, operators. To verify the remaining claims, first of all we note that, by construction,

$$
K x_{j}=\sum_{i=1}^{j} c_{i j} x_{i}, j \geq 1
$$

with $c_{i j} \in \mathbb{F}$ and $c_{j j} \neq 0$. Thus

$$
K \mathcal{V} \supseteq\left\langle x_{j}\right\rangle_{j=1}^{\infty}
$$

On the other hand, since $T_{j}^{\prime}(x)=0$ for all $x \in \mathcal{V}$ and all positive integers $j$, and since $\mathcal{V}$ is closed, it easily follows from the series defining $K$ that $K x \in \mathcal{V}$ for all $x \in \mathcal{V}$. Thus $K \mathcal{V} \subseteq \mathcal{V}$. So we can write

$$
\left\langle x_{j}\right\rangle_{j=1}^{\infty} \subseteq K \mathcal{V} \subseteq \mathcal{V}
$$

Thus

$$
\mathcal{V}=\overline{\left\langle x_{j}\right\rangle_{j=1}^{\infty}} \subseteq \overline{K \mathcal{V}} \subseteq \overline{\mathcal{V}}=\mathcal{V}
$$

Hence $\overline{K \mathcal{V}}=\mathcal{V}$.

It follows from the series defining $K$ that

$$
K y_{j}=x_{j}^{\prime}+\sum_{i=1}^{j} c_{i j}^{\prime} y_{i}, j \geq 1
$$

with $c_{i j}^{\prime} \in \mathbb{F}$ and $c_{j j}^{\prime} \neq 0$ and where $x_{j}^{\prime} \in \mathcal{V}=\overline{K \mathcal{V}}$. Thus $\overline{K X} \supseteq\left\langle y_{j}\right\rangle_{j=1}^{\infty}$. Obviously $\overline{K X} \supseteq \overline{K \mathcal{V}}=\mathcal{V}$. Hence

Therefore

$$
\overline{K X} \supseteq\left\langle\left\langle y_{j}\right\rangle_{j=1}^{\infty}, \mathcal{V}\right\rangle .
$$

$$
\overline{K X} \supseteq\left\{x_{i}\right\}_{i=1}^{\infty} \cup\left\{y_{i}\right\}_{i=1}^{\infty} .
$$


So

$$
\overline{K X} \supseteq \overline{\left\langle\left\{x_{i}\right\}_{i=1}^{\infty} \cup\left\{y_{i}\right\}_{i=1}^{\infty}\right\rangle}=X
$$

i.e., the range of $K$ is dense in $X$. We note that in the proof above we assumed that both $\mathcal{V}$ and $X / \mathcal{V}$ are infinite-dimensional. If either $\mathcal{V}$ or $X / \mathcal{V}$ is finite-dimensional, we end up having finitely many of either $x_{i}$ 's or $y_{i}$ 's in which case the proof can easily be adjusted. This completes the proof.

(b) Let $\mathcal{V} \subseteq X$ be given. Consider $\mathcal{V}^{\perp}=\left\{f \in X^{*}: f(\mathcal{V})=0\right\}$. Note that $\mathcal{V}^{\perp}$ is a weak* closed, thus a norm closed, subspace of $X^{*}$. Also note that $X^{*}$ is $\mathrm{w}^{*}$-separable by Proposition 1.3 Let $\left\{f_{i}\right\}_{i=1}^{\infty}$ be a $\mathrm{w}^{*}$-dense subset of $\mathcal{V}^{\perp}$. Enlarge $\left\{f_{i}\right\}_{i=1}^{\infty}$ to a $\mathrm{w}^{*}$-dense subset $\left\{f_{i}\right\}_{i=1}^{\infty} \cup\left\{g_{i}\right\}_{i=1}^{\infty}$ of $X^{*}$. With no loss of generality, we may assume that $f_{i} \neq 0$ and $g_{i} \notin \mathcal{V}^{\perp}$ for all $i \in \mathbb{N}$. If necessary, by choosing subsequences of $\left\{f_{i}\right\}_{i=1}^{\infty}$ and $\left\{g_{i}\right\}_{i=1}^{\infty}$, we may suppose that $\left\{f_{i}\right\}_{i=1}^{\infty} \cup\left\{g_{i}\right\}_{i=1}^{\infty}$ is independent, $\overline{\left\langle\left\{f_{i}\right\}_{i=1}^{\infty}\right\rangle}{ }^{w *}=\mathcal{V}^{\perp}$, and that $\overline{\left\langle\left\{f_{i}\right\}_{i=1}^{\infty} \cup\left\{g_{i}\right\}_{i=1}^{\infty}\right\rangle}{ }^{w *}=X^{*}$.

Let $x_{1} \in X$ be such that $f_{1}\left(x_{1}\right) \neq 0$. Set $S_{1}:=f_{1} \otimes x_{1}$ defined on $X^{*}$ by $S_{1} g=g\left(x_{1}\right) f_{1}$. We take notice of the fact that $S_{1}=T_{1}^{*}$ where $T_{1}=x_{1} \otimes f_{1}$ defined on $\mathcal{X}$ by $T_{1} x=f_{1}(x) x_{1}$, and that $\left\|S_{1}\right\|=\left\|T_{1}\right\|$. It follows from Lemma 1.1 that Ker $f_{1} \not \subseteq$ Ker $f_{2}$. Thus there exists $x_{2} \in X$ such that $f_{1}\left(x_{2}\right)=0$ but $f_{2}\left(x_{2}\right) \neq 0$. Set $S_{2}=f_{2} \otimes x_{2}$. As before take notice of the fact that $S_{2}=T_{2}^{*}$ where $T_{2}=x_{2} \otimes f_{2}$, and that $\left\|T_{2}\right\|=\left\|S_{2}\right\|$. Now suppose that $x_{j}$ 's $(1 \leq j \leq k-1)$ are chosen. Again it

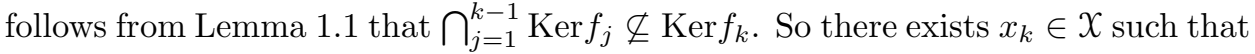
$f_{j}\left(x_{k}\right)=0$ for all $1 \leq j \leq k-1$, but $f_{k}\left(x_{k}\right) \neq 0$. Set $S_{k}=f_{k} \otimes x_{k}$ and note that $S_{k}=T_{k}^{*}$ where $T_{k}=x_{k} \otimes f_{k}$, and that $\left\|T_{k}\right\|=\left\|S_{k}\right\|$. Note that $S_{k} f_{j}=f_{j}\left(x_{k}\right) f_{k}$; thus $S_{k} f_{j}=0$ for $k>j$ and $S_{k} f_{k}=f_{k}\left(x_{k}\right) f_{k} \neq 0$.

Since $g_{1} \notin \mathcal{V}^{\perp}$, it follows from the definition that there exists $y_{1} \in \mathcal{V}$ such that $g_{1}\left(y_{1}\right) \neq 0$. Set $S_{1}^{\prime}=g_{1} \otimes y_{1}$ and notice that $S_{1}^{\prime}=T_{1}^{\prime *}$ where $T_{1}^{\prime}=y_{1} \otimes g_{1}$, and that $\left\|T_{1}^{\prime}\right\|=\left\|S_{1}^{\prime}\right\|$. Suppose that $y_{j}$ 's $(1 \leq j \leq k-1)$ are chosen. Since $g_{k} \notin$ $\left\langle g_{1}, \ldots, g_{k-1}, \mathcal{V}^{\perp}\right\rangle$, it follows from Corollary 1.2 that there is $y_{k} \in \mathcal{V} \cap\left(\bigcap_{j=1}^{k-1} \operatorname{Ker} g_{j}\right)$ such that $g_{k}\left(y_{k}\right) \neq 0$. Set $S_{k}^{\prime}=g_{k} \otimes y_{k}$ and notice that $S_{k}^{\prime}=T_{k}^{\prime *}$ where $T_{k}^{\prime}=y_{k} \otimes g_{k}$, and that $\left\|T_{k}^{\prime}\right\|=\left\|S_{k}^{\prime}\right\|$. We note that $S_{k}^{\prime} f=f\left(y_{k}\right) g_{k}=0$ for all $f \in \mathcal{V}^{\perp}$. In particular, $S_{k}^{\prime} f_{j}=0$ for all $k, j \geq 1$, and that $S_{k}^{\prime} g_{j}=g_{j}\left(y_{k}\right) g_{k}$; thus $S_{k}^{\prime} g_{j}=0$ for $k>j$ and $S_{k}^{\prime} g_{k}=g_{k}\left(y_{k}\right) g_{k} \neq 0$.

Define

$$
S:=\sum_{j=1}^{\infty}\left(\frac{S_{j}}{2^{j}\left\|S_{j}\right\|}+\frac{S_{j}^{\prime}}{2^{j}\left\|S_{j}^{\prime}\right\|}\right)
$$

Note that $S=T^{*}$ where

$$
T=\sum_{j=1}^{\infty}\left(\frac{T_{j}}{2^{j}\left\|T_{j}\right\|}+\frac{T_{j}^{\prime}}{2^{j}\left\|T_{j}^{\prime}\right\|}\right) .
$$

The operators $S$ and $T$ are compact since they are norm limits of finite rank operators. We claim that the range of $S$ is $\mathrm{w}^{*}$-dense in $X^{*}$ and that ${\overline{S \mathcal{V}^{\perp}}}^{w *}=\mathcal{V}^{\perp}$.

Now, we note that by construction we can write

$$
S f_{j}=\sum_{i=1}^{j} c_{i j} f_{i}, j \geq 1
$$

with $c_{i j} \in \mathbb{F}$ and $c_{j j} \neq 0$. Thus

$$
S V^{\perp} \supseteq\left\langle f_{j}\right\rangle_{j=1}^{\infty} .
$$


Since $S=T^{*}, S$ is $\mathrm{w}^{*}$ to $\mathrm{w}^{*}$ continuous by Theorem 3.1.11 on page 287 of [M]. Let $h \in \mathcal{V}^{\perp}$ be an arbitrary element. Since $\left\langle f_{i}\right\rangle_{i=1}^{\infty}$ is $\mathrm{w}^{*}$-dense in $\mathcal{V}^{\perp}$, it follows that $h=\mathrm{w}^{*}-\lim _{j} h_{j}$ for some sequence $\left\{h_{j}\right\}_{j=1}^{\infty}$ in $\left\langle f_{i}\right\rangle_{i=1}^{\infty}$. Now since $S$ is w* to w* continuous, it follows that $S h=\mathrm{w}^{*}-\lim _{j} S h_{j}$ but $S h_{j} \in \mathcal{V}^{\perp}$ by the series defining $S$ (note that $S_{k}^{\prime} f=f\left(y_{k}\right) g_{k}=0$ for all $f \in \mathcal{V}^{\perp}$, and that $\mathcal{V}^{\perp}$ is $\mathrm{w}^{*}$-closed). Therefore $S h \in \mathcal{V}^{\perp}$. So since $h$ was arbitrary, we conclude that

$$
\left\langle f_{i}\right\rangle_{i=1}^{\infty} \subseteq S V^{\perp} \subseteq \mathcal{V}^{\perp}
$$

Hence

$$
\mathcal{V}^{\perp}={\overline{\left\{f_{i}\right\}_{i=1}^{\infty}}}^{w *} \subseteq{\overline{S \mathcal{V}^{\perp}}}^{w *} \subseteq{\overline{\mathcal{V}^{\perp}}}^{w *}=\mathcal{V}^{\perp}
$$

implying that ${\overline{S V^{\perp}}}^{w *}=\mathcal{V}^{\perp}$. Now it follows from the series defining $S$ that

$$
S g_{j}=f_{j}^{\prime}+\sum_{i=1}^{j} c_{i j}^{\prime} g_{i}, j \geq 1
$$

with $c_{i j}^{\prime} \in \mathbb{F}$ and $c_{j j}^{\prime} \neq 0$, where $f_{j}^{\prime} \in \mathcal{V}^{\perp}={\overline{S \mathcal{V}^{\perp}}}^{w *}$ (note that $f_{j}^{\prime} \in \mathcal{V}^{\perp}$ follows from the fact that $\mathcal{V}^{\perp}$ is norm closed). Thus ${\overline{S X^{*}}}^{w *} \supseteq\left\langle g_{j}\right\rangle_{j=1}^{\infty}$. Obviously

$$
{\overline{S X^{*}}}^{w *} \supseteq{\overline{S \mathcal{V}^{\perp}}}^{w *}=\mathcal{V}^{\perp} \text {. }
$$

It follows that

So we can write

$$
{\overline{S X^{*}}}^{w *} \supseteq\left\langle\left\langle g_{j}\right\rangle_{j=1}^{\infty}, \mathcal{V}^{\perp}\right\rangle
$$

$$
{\overline{S X^{*}}}^{w *} \supseteq\left\{f_{i}\right\}_{i=1}^{\infty} \cup\left\{g_{i}\right\}_{i=1}^{\infty} .
$$

Thus

$$
{\overline{S X^{*}}}^{w *} \supseteq{\overline{\left\langle\left\{f_{i}\right\}_{i=1}^{\infty} \cup\left\{g_{i}\right\}_{i=1}^{\infty}\right\rangle}}^{w *}=X^{*},
$$

i.e., ${\overline{T^{*} X^{*}}}^{w *}=X^{*}$. So by Theorem 3.1.17 of $[\mathrm{M}], T$ is $1-1$.

That $T$ leaves $\mathcal{V}$ invariant is not that difficult to see. We have $T_{k} x=f_{k}(x) x_{k}$, and so for all $x \in \mathcal{V}$ and for all $k \geq 1$, we have $T_{k} x=0$. Now letting $x \in \mathcal{V}$ be arbitrary, we can write

$$
T x=\sum_{j=1}^{\infty} \frac{T_{j} x}{2^{j}\left\|T_{j}\right\|}+\frac{T_{j}^{\prime} x}{2^{j}\left\|T_{j}^{\prime}\right\|}=\sum_{j=1}^{\infty} \frac{g_{j}(x) y_{j}}{2^{j}\left\|T_{j}^{\prime}\right\|} \in \mathcal{V},
$$

for $y_{k} \in \mathcal{V}, k \in \mathbb{N}$ and note that $\mathcal{V}$ is a closed subspace of $X$. Thus $T \mathcal{V} \subseteq \mathcal{V}$. So $T: X \longrightarrow X$ is compact, $1-1$, and leaves $\mathcal{V}$ invariant. Again in the proof above we assumed that both $\mathcal{V}^{\perp}$ and $X^{*} / \mathcal{V}^{\perp}$ are infinite-dimensional. If either of them is finite-dimensional, just as we mentioned in the proof of part (a), we end up having finitely many $f_{i}$ 's or $g_{i}$ 's, in which case the proof can easily be adjusted.

Remark. For a given subspace $\mathcal{V}$ of a (real or complex) Banach space $\mathcal{X}$, we do not know whether there exists a one-to-one compact operator $K$ in the norm closure of finite-rank operators on $X$ whose range is dense and such that $\overline{K \mathcal{V}}=\mathcal{V}$.

Theorem 2.2. Let $X$ be an infinite-dimensional separable Banach space, and let $\mathcal{F}$ be a finite chain of subspaces.

(a) There exists a compact operator $K$ in the norm closure of finite-rank operators on $\mathcal{X}$ whose range is dense and such that $\overline{K \mathcal{V}}=\mathcal{V}$ for all $\mathcal{V} \in \mathcal{F}$.

(b) There exists a one-to-one compact operator $K$ in the norm closure of finiterank operators on $\mathcal{X}$ that leaves $\mathcal{V}$ invariant for all $\mathcal{V} \in \mathcal{F}$. 
Proof. (a) Suppose that

$$
\mathcal{V}_{0}:=\{0\} \subset \mathcal{V}_{1} \subset \ldots \subset \mathcal{V}_{n} \subset \mathcal{V}_{n+1}:=X
$$

is a given finite chain of subspaces. It is easily seen that there is a countable independent set

$$
\bigcup_{i=1}^{n+1}\left\{x_{i j}\right\}_{1 \leq j \leq \operatorname{dim}\left(\mathcal{V}_{i} / \mathcal{v}_{i-1}\right)}
$$

of elements of $\mathcal{X}$ such that $\bigcup_{i=1}^{k}\left\{x_{i j}\right\}_{1 \leq j \leq \operatorname{dim}\left(\mathcal{V}_{i} / \mathcal{V}_{i-1}\right)}$ is dense in $\mathcal{V}_{k}$ for all $1 \leq k \leq$ $n+1$ and such that $x_{i j} \notin \mathcal{V}_{i-1}$ for all $1 \leq i \leq n+1,1 \leq j \leq \operatorname{dim}\left(\mathcal{V}_{i} / \mathcal{V}_{i-1}\right)$. The rest of the proof is similar to that of Theorem 2.1. We omit the details.

(b) Just as in the proof of the (a) part of the theorem, the idea of the proof of part (b) is identical to that of Theorem 2.1(b).

Remark. 1. Again if the underlying space happens to be a real or complex Hilbert space, then, just as we saw in the remark preceding the proof of Theorem 2.1 a similar argument shows that there exists a one-to-one compact normal operator $K$ whose range is dense and such that $\overline{K \mathcal{V}}=\mathcal{V}$ for all $\mathcal{V} \in \mathcal{F}$ where $\mathcal{F}$ is the given finite chain of subspaces.

2. Similarly, by adjusting the proof of part (a) of Theorem 2.2, it is not difficult to see that for a given countable chain $\mathcal{F}$ of subspaces of an infinite-dimensional separable Banach space $X$, of the form

$$
\mathcal{V}_{0}:=\{0\} \subset \mathcal{V}_{1} \subset \ldots \subset \mathcal{V}_{n} \subset \ldots \subset \mathcal{V}_{\infty}:=X,
$$

there always exists a compact operator $K$ in the norm closure of finite-rank operators whose range is dense and such that $\overline{K \mathcal{V}}=\mathcal{V}$ for all $\mathcal{V} \in \mathcal{F}$.

Theorem 2.3. Let $\mathcal{X}$ be an infinite-dimensional separable Banach space, and $\mathcal{S}$ a semigroup of operators in $\mathcal{B}(\mathcal{X})$. Then the following are equivalent:

(i) The semigroup $\mathcal{S}$ is reducible.

(ii) There exists a one-to-one compact operator $K$ in the norm closure of finiterank operators such that the collection

$$
\mathcal{S} K=\{A K: A \in \mathcal{S}\}
$$

is reducible.

(iii) There exists a compact operator $K$ in the norm closure of finite-rank operators whose range is dense and such that the collection

$$
K \mathcal{S}=\{K A: A \in \mathcal{S}\}
$$

is reducible.

Proof. Let $\mathcal{A}$ denote the algebra generated by the semigroup $\mathcal{S}$. We note that $\mathcal{A}=\langle\mathcal{S}\rangle$. That being noted, it suffices to prove the assertion for an algebra $\mathcal{A}$ of operators in $\mathcal{B}(\mathcal{X})$. We prove that $(i) \Longleftrightarrow(i i)$ and $(i) \Longleftrightarrow(i i i)$.

$(i) \Longrightarrow($ ii $)$ Suppose that $\mathcal{A}$ is reducible. So there exists a nontrivial subspace $\{0\} \supsetneqq \mathcal{V} \supsetneqq X$ such that $\mathcal{A V} \subseteq \mathcal{V}$. By Theorem 2.1(b), there exists a one-to-one compact operator $K$ such that $K \mathcal{V} \subseteq \mathcal{V}$. It is plain that $\mathcal{A} K \mathcal{V} \subseteq \mathcal{V}$.

$($ i $) \Longrightarrow($ iii $)$ Can be proven in a similar manner as in $(i) \Longrightarrow($ ii $)$ except that one has to use Theorem 2.1 (a).

(ii) $\Longrightarrow(i)$ Let $\{0\} \supsetneqq \mathcal{V} \varsubsetneqq \mathcal{X}$ be a nontrivial subspace of $\mathcal{X}$ such that $\mathcal{A} K \mathcal{V} \subseteq \mathcal{V}$ where $K$ is a $1-1$ compact operator. Choose $0 \neq x \in \mathcal{V}$ and set $\mathcal{W}:=\overline{\mathcal{A} K x}$. If 
$\mathcal{W}=0$, then $\{0\} \supsetneqq\langle K x\rangle \supsetneqq X$, and $\langle K x\rangle$ is a nontrivial closed subspace of $\mathcal{A}$. If $\mathcal{W} \neq 0$, then $\{0\} \supsetneqq \mathcal{W} \leq \mathcal{V} \supsetneqq X$ is obviously a nontrivial closed subspace of $\mathcal{A}$. So in any event, $\mathcal{A}$ is reducible.

$($ iii $) \Longrightarrow(i)$ Let $\{0\} \supsetneqq \mathcal{V} \supsetneqq X$ be a nontrivial subspace of $X$ such that $K \mathcal{A} \mathcal{V} \subseteq \mathcal{V}$ where $K$ is a compact operator whose range is dense. Choose $0 \neq x \in \mathcal{V}$ and set $\mathcal{W}:=\overline{\mathcal{A} x}$. If $\mathcal{W}=0$, then $\langle x\rangle$ is a closed nontrivial subspace of $\mathcal{A}$. If $\mathcal{W} \neq 0$, then $\{0\} \supsetneqq \mathcal{W} \supsetneqq X$ is obviously a nontrivial closed subspace of $\mathcal{A}$. That $\mathcal{W} \supsetneqq X$ follows from the fact that $K$ has dense range and that $K \overline{\mathcal{A} x} \subseteq K \overline{\mathcal{A V}} \subseteq \overline{K \mathcal{A V}} \subseteq \overline{\mathcal{V}} \subseteq \mathcal{V}$. So in any event $\mathcal{A}$ is reducible.

Remark. 1. Let $\mathcal{S}$ be as in Theorem 2.3. and $A, B \in \mathcal{B}(X)$ where $A$ is $1-1$ and $B$ is a dense-range bounded operator. It follows from the proof of the preceding theorem that $\mathcal{S}$ is reducible if $\mathcal{S} A$ or $B \mathcal{S}$ is reducible.

2. It is shown in [Y1] (see Theorem 4.2 of [Y1]) that triangularizability of $S A$ implies reducibility of $\mathcal{S}$ provided that $\operatorname{rank}(A) \geq 2$. As pointed out in the remark following Theorem 4.2 of [Y1], the assertion we just mentioned holds on finitedimensional vector spaces over general fields.

3. By Lemma 2.5.1 of $\bar{Y} 2$, reducibility of $T \mathcal{S} \mid \overline{T x}$ implies that of the semigroup $\mathcal{S}$ provided that $T \neq 0$. The finite-dimensional counterpart of this lemma is Lemma 2.2.1 of [Y2] which says: Let $\mathcal{V}$ be a finite-dimensional vector space over a field $F$, and $\mathcal{S}$ a semigroup in $\mathcal{L}(\mathcal{V})$, and $T$ a nonzero linear transformation in $\mathcal{L}(\mathcal{V})$. If $\mathcal{S}$ is irreducible, then so is $\left.T \mathcal{S}\right|_{\mathcal{R}}$ where $\mathcal{R}=T \mathcal{V}$ is the range of $T$.

We conclude with the following questions, which we have not been able to resolve.

Let $\mathcal{X}$ be an infinite-dimensional separable real or complex Banach space, and $\mathrm{C}$ an arbitrary chain of subspaces.

1. Does there exists a compact operator $K$ on $X$ whose range is dense and such that $\overline{K \mathcal{V}}=\mathcal{V}$ for all $\mathcal{V} \in \mathcal{C}$ ?

2. Does there exists a one-to-one compact operator $K$ on $X$ that leaves the chain $\mathcal{C}$ invariant, i.e., $K \mathcal{V} \subseteq \mathcal{V}$ for all $\mathcal{V} \in \mathcal{C}$ ?

Note that since every chain of subspaces can be extended to a maximal chain of subspaces, it suffices to answer the above questions for maximal chains of subspaces.

\section{ACKNOWLEDGMENT}

I would like to thank my supervisor Professor Heydar Radjavi for his encouragement and very helpful comments. I would also like to thank the referee for the helpful comments.

\section{REFERENCES}

[C] J. B. Conway, A Course in Functional Analysis, Springer-Verlag, New York, 1990. MR 91e:46001

[M] R. E. Megginson, An Introduction to Banach Space Theory, Springer-Verlag, New York, 1998. MR 99k:46002

[RR] H. Radjavi and P. Rosenthal, Simultaneous Triangularization, Springer-Verlag, New York, 2000. MR 2001e:47001 
[Y1] B. R. Yahaghi, Near triangularizability implies triangularizability, to appear in the Canadian Mathematical Bulletin.

[Y2] B. R. Yahaghi, Reducibility Results on Operator Semigroups, Ph.D. Thesis, Dalhousie University, Halifax, Canada, 2002.

Department of Mathematics, Univerity of Toronto, Toronto, Ontario, Canada M5S $3 \mathrm{G} 3$

E-mail address: bamdad5@math.toronto.edu

E-mail address: reza5@mscs.dal.ca 